\title{
Is maintenance iron dosing associated with a lower infection risk than bolus dosing in haemodialysis patients?
}

Results of a new study suggest that administering iron to patients on haemodialysis in regular maintenance doses may lead to fewer infections than giving iron in larger doses on an as-needed basis.

Anaemia is common in patients on haemodialysis and is usually managed with erythropoiesis-stimulating agents (ESAs) and intravenous iron supplementation. Safety concerns regarding ESAs have led to increased use of intravenous iron, but intravenous iron carries concerns of infection risk. Iron can be given in large doses on an as-needed basis (bolus dosing) or it can be given in low doses every 1-2 weeks to maintain iron stores (maintenance dosing).

Brookhart et al. used data from 117,050 dialysis patients in the USA merged with Medicare data to investigate the associations between dosing patterns in 1-month exposure periods and infection-related hospitalization over the following 3 months. Overall, $49 \%$ of patients received maintance dosing of iron, $13 \%$ received bolus dosing of iron and $38 \%$ received no iron.

\section{4 ...the role of iron in bacterial growth and infection risk is biologically plausible and has been shown in animal models... 77}

The researchers found that patients who received bolus dosing of iron were at higher risk of infection-related hospitalization during follow-up compared with those receiving maintenance iron dosing (risk difference 25 additional events per 1,000 patientyears). Infection risk was highest in patients with a catheter and in those with a history of a recent infection. Maintenance iron dosing was not associated with an increased risk of adverse outcomes compared with no iron.

"Although the role of iron in bacterial growth and infection risk is biologically plausible and has been shown in animal models, data from large CKD [chronic kidney disease] populations on iron and infections are lacking," say the authors. They say that their results suggest that bolus dosing of iron may increase the short-term risk of infection-related hospitalization, but that the potential infection risk associated with more aggressive iron use must be balanced with its known benefits, such as diminished ESA requirements and improved anaemia management.

Rebecca Kelsey

Original article Brookhart, M. A. et al. Infection risk
with bolus maintenance iron supplementation in
hemodialysis patients. J. Am. Soc. Nephrol. doi:10.1681/
ASN.2012121164

